\title{
Control loops with detection of inner electrical resistance and fatigue-behaviour by activation of $\mathrm{NiTi}$-Shape Memory Alloys
}

\author{
H. Meier, A. Czechowicza, C. Haberland \\ Ruhr-Universität Bochum, \\ Lehrstuhl für Produktionssysteme, 44801 Bochum, Germany
}

\begin{abstract}
Shape memory alloys are smart materials. Due to their ability to change into a previously imprinted actual shape through the means of thermal activation, they are suitable as actuators for microsystems and, within certain limitations, macroscopic systems. To apply these smart materials to a wide range of industrial applications, a simple method of controlling the actuator effect is required. The detection of inner electrical resistance allows to gauge the actuator movement. By usage of a microcontroller a smart system without any hardware sensors can be realized. Changing outer boundary conditions can be compensated by software. Furthermore an analysis and a description of the functional fatigue, affecting the control loop, is of particular importance. A fatigue calculator dependent upon duty cycle is subjoined to the existing actuator simulation implemented in MATLAB/ SIMULINK. The focus of the simulation-model is on the activation behaviour of the SMA actuator, which defines its rate of heating and cooling. These parameters describe the dynamical characteristics of the actuator and the complete SMA powered system. Different load conditions, various actuator geometries and shapes, e.g. wire or spring actuator are simulated by the calculation of the energetic balance of the whole system. The numerical model can be used to simulate time variant heating currents in order to achieve an optimal system performance for a defined time response of the actuator.
\end{abstract}

\section{Shape memory actuators and their importance for mechatronical systems}

Shape memory alloys (SMAs) exhibit the remarkable property to recover a previously imprinted shape after a deformation. That effect can occur either at constant temperatures (mechanical memory, pseudoelasticity), or changing temperatures (thermal memory). Both effects rely on the martensitic phase transformation [1]. During heating the transformation from the low temperature phase B19' (martensite) into B2 (austenite) initiates by exceeding the austenite start temperature. This is linked to a shape change. During subsequent cooling the reconversion of the material is provoked by the retransformation B2 into B19'. This work focuses on the one way effect in combination with restoring force devices, the so called extrinsic two way effect [2].

Most commonly used shape memory materials are binary nickel titanium alloys (NiTi), consisting nearly of a $50 \%$ ratio of both elements. The ratio is the crucial factor for setting up transformation temperatures. Shape memory alloys have certain characteristics which are unique in comparison to other actuating principles. Today's competitiveness of micro- and mechatronical systems is determined by precision, cost, quality and simplifications. Systems driven by shape memory elements can be used for smart actuators, which include aspects of flexibility and mass decimation in addition to the above-qouted. The aspect of simplification is related to the system's costs, because a shape memory actuator generally consists of fewer parts than conventional ones. The reduction of parts often enhances the reliability of actuating components. Besides the mass of shape memory systems, as compared to other actuators, is lower.). A striking advantage of SMAs is the significant higher working capacity in comparison to conventional actuators[3].

In terms of designing smart actuator systems, SMAs provide the possibility to create controller systems without sensor hardware. The detection of inner electrical resistance allows to steer the actuator movement [4]. For example a simple valve component with NiTi drive activated by external heating can be used as well as an electrically activated valve or a controlled proportional valve. This also decreases costs as the volume of systems variety can be reduced.

Nowadays many researchers focus their work on applying NiTi to actuator applications, but

a e-mail: Czechowicz@lps.rub.de

This is an Open Access article distributed under the terms of the Creative Commons Attribution-Noncommercial License (http://creativecommons.org/licenses/by-nc/3.0/), which permits unrestricted use, distribution, and reproduction in any noncommercial medium, provided the original work is properly cited. 
fundamental problems for their commercial use still remain. This includes control paradigms and functional fatigue effects [1]. Hence the present work deals with concepts, resolving these problems by integration of an appropriate calculation into a numerical simulation of the activation behavior of SMA wire actuators. For that purpose analysis and modeling of electrical resistance behavior aims for enhancement of an SMA numerical simulation of controlled systems. Furthermore a life cycle calculation based on a fatigue simulation is implemented in the model, too.

\section{Experimental}

\subsection{Analysis of electrical resistance}

As already described by Ikuta the most interesting aspect regarding electrical resistance of NiTi is the applicability of detecting actuator positions [4]. However the electrical resistance behavior of SMAs differs from common metals. The electrical resistance of metals mainly depends on the temperature and their dimension. Exemplary the stretching of a metal increases its resistance.

SMAs exhibit different resistances depending on their structure. Thus the resistance of the B19' phase is higher than resistance of B2 phase [5]. For example the specific resistance of SMA wire can be described as a function of the phase transformation.

$$
\begin{gathered}
R_{A}=\rho_{N i T i} \cdot \frac{l_{w}}{A_{w}} \cdot\left[\alpha_{N i T i} \cdot \Delta T+1\right] \\
\text { and: } \\
\rho_{N i T i}=\rho_{\text {aus }} \cdot \psi(T)+\rho_{\text {mar }} \cdot[1-\psi(T)] \\
\text { with: }
\end{gathered}
$$

$R_{A}=$ electrical resistance

$A_{w}=$ wire-actuator aperture

$l_{w}=$ wire-actuator length

$T=$ temperature

$\alpha_{N i T i}=$ thermal resistance coefficient for NiTi

$\psi=$ volume ratio of the $B 2$ phase $(0<\psi<1)$

$\rho_{\text {NiTi }}=$ specific electrical resistance of NiTi

$\rho_{\text {NiTi }}=\rho_{\text {mar }}=1,067 \cdot 10^{-6} \Omega \mathrm{mm}$ for $\psi=0$

$\rho_{\text {NiTi }}=\rho_{\text {aus }}=1,014 \cdot 10^{-6} \Omega \mathrm{mm}$ for $\psi=1$

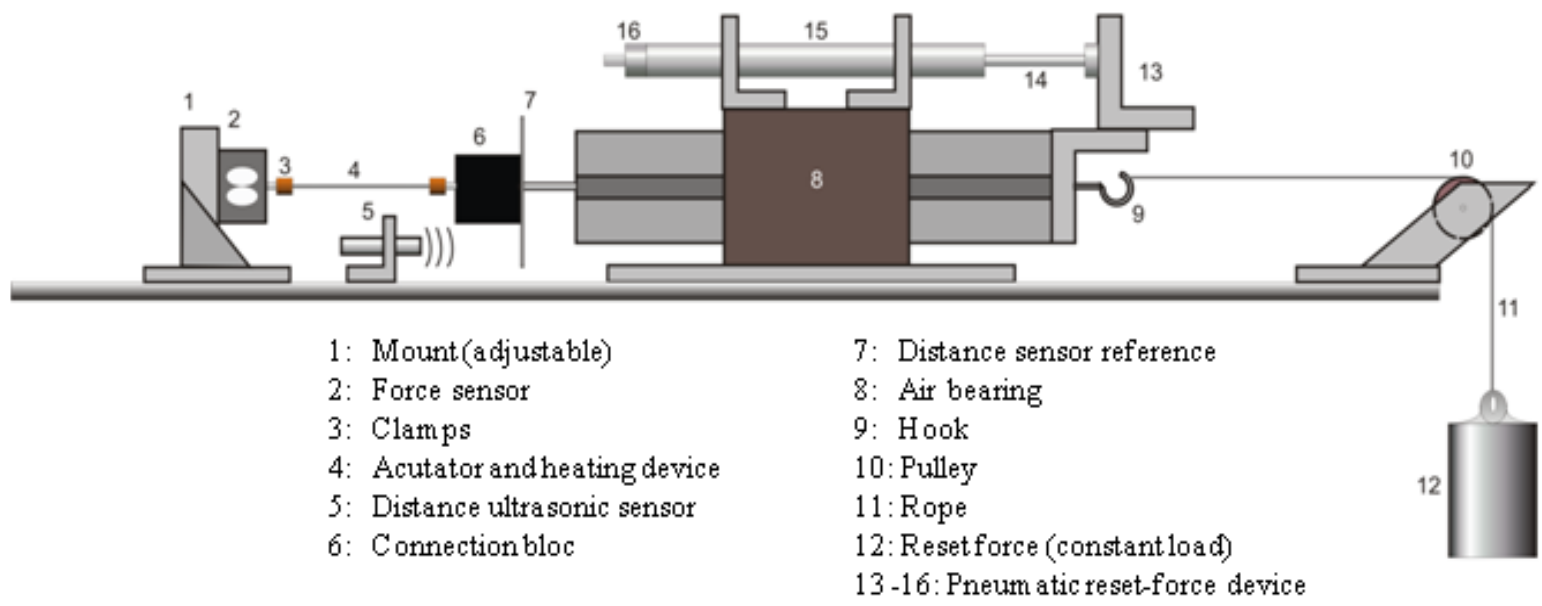

Fig.1. Test stand for shape memory wires 
The thermal resistance coefficient $\alpha_{N i T i}$ has to be identified with experimental data. For that purpose a test stand with an integrated thermal controlled field was developed (figure 1). Within this stand a SMA wire actuator is joined with a frictionless and programmable restoring force device. The wire is activated by an external heating unit. An ultrasonic distance sensor detects the wire stroke. During measurement a constant current of $50 \mathrm{~mA}$ was used as sensoring signal. In passing it is noted that this current is too low for provoking the phase transformation. During testing the temperature was increased from $20^{\circ} \mathrm{C}$ to $150^{\circ} \mathrm{C}$ by the heating device. Thus the output signal, voltage, varies. As the total electrical resistance can be calculated by Ohm's law, the thermal resistance coefficient can be calculated from equation 1 .

Figure 2 exemplary shows the characteristic of the phase transformation by the electrical resistance plotted against the stroke of the wire. This characteristic can be subdivided into three parts. In the first part, the material consists of pure martensite. During transformation, the phase-dependant decrease of the specific electrical resistance $\left(\rho_{\mathrm{NiTi}}\right)$ is major to the increases caused by the thermal resistance change $\left(\alpha_{N i T i}\right)$ This causes a decline of the absolute resistance in second part. The phase transformation is finished at the end of the second part, so the material consists completely of austenite. The resistance increase of the third phase is characterized by conventional metallic resistance change depending on temperature.

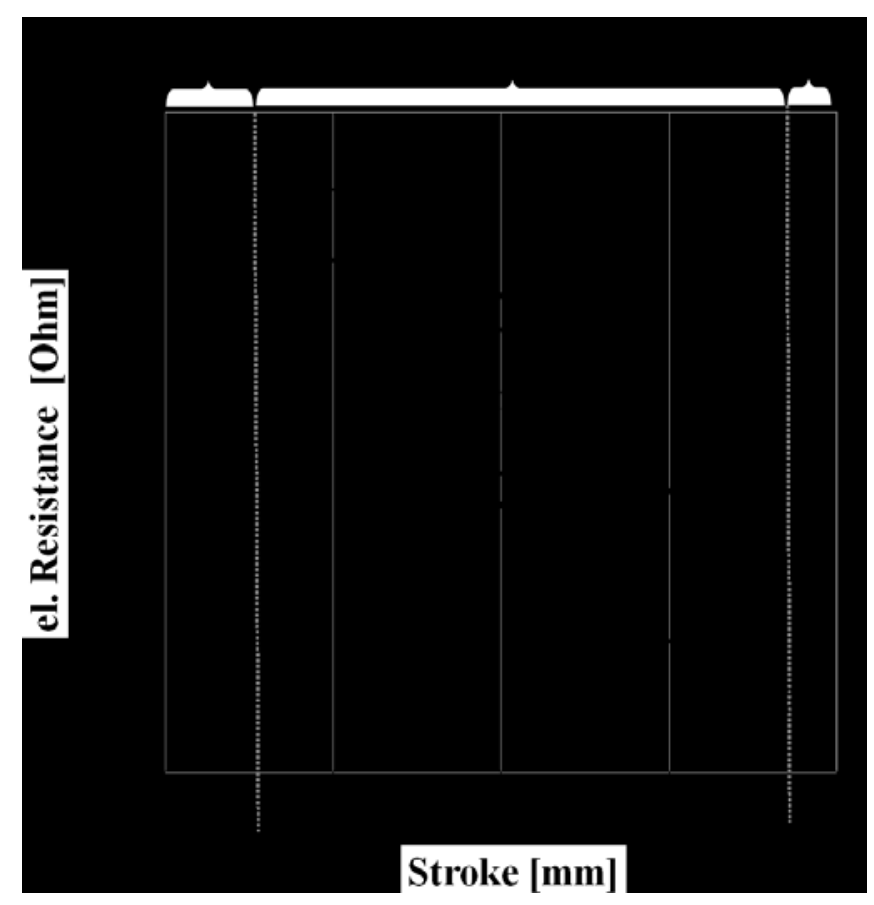

Fig.2. Resistance hysteresis of a NiTi SMA 


\section{Resistance- controlled shape memory systems}

\subsection{Numerical Simulation of shape memory actuators}

Try and test methods are often used to appraise the system behavior of shape memory actuators. It is well known that such methods often slow down the engineering process and can cost quite a lot. A numerical simulation developed by the authors can calculate the activation behavior of NiTi wire-, flat wire- and spring actuators. The user can choose the actuator type, the loads and the boundary conditions (temperature, alloy type, current, etc.) at start. The system response, calculated by the numerical model, can be used to design actuator systems. Figure 3 shows the simulated activation behavior in comparison to a real system. As shown in figure 3 there is a slight discrepancy between both curves what can be explained either by the influences of the test stand (e.g. mass effects) or the simplifications programmed in the simulation. For example heat transfer mechanisms from the actuator to the closest environment are not implemented in the numerical model so far. On the other side, the achievement of the maximal stroke is of major importance for mechatronical applications, so the simulation delivers usable data.

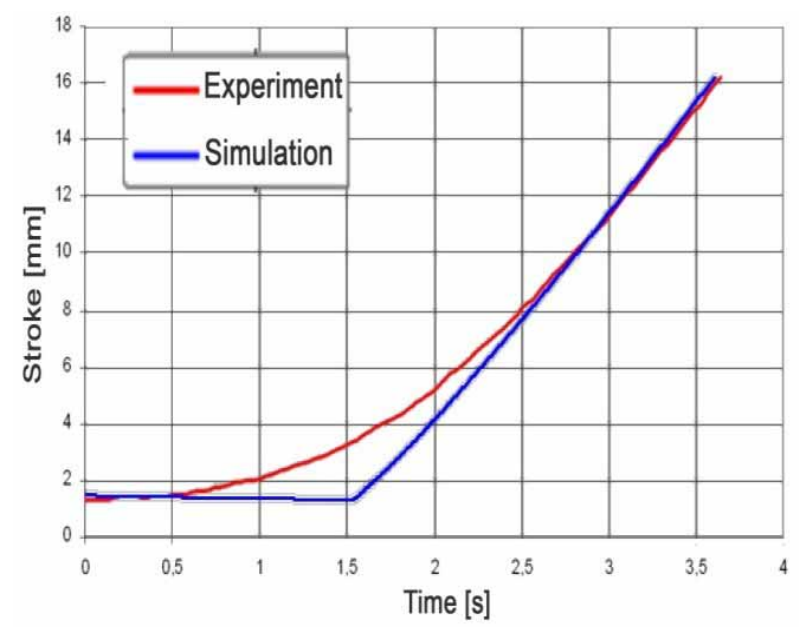

Fig. 3. Numerical Simulation of SMA activation

Using the interrelationship of phase transformation, boundary conditions and the physical coherences in equation 1 the simulation of the resistance behavior has been implemented in a numerical model. The model provides a characteristic similar to the real experiment (figure 4). The hysteresis- and the total resistance values are prognosed correctly. The differences between the real and the simulated resistance, especially at the curve's endpoints, result from the simplifications of boundary conditions. The transformation is simply described in the hysterical model by the law of cosinus. This approach done by Liang and Rogers [6] and enhanced by Oelschläger [2] is an approximation towards the real phase transformation of NiTi. 


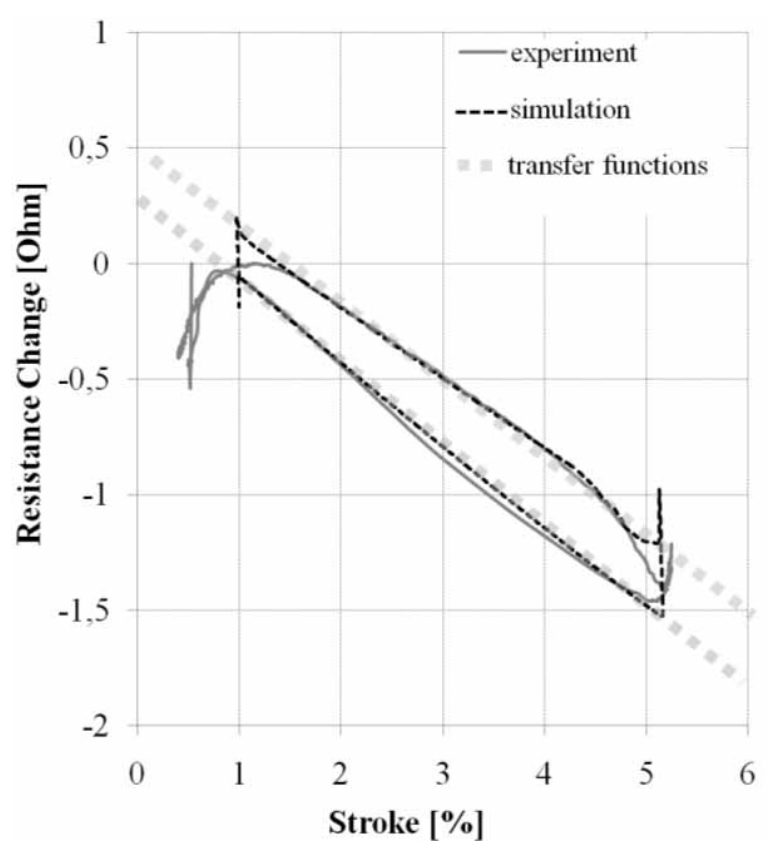

Fig. 4. SMA simulation of resistance curves

\subsection{Programming and calibration of control loops with resistance detection}

The approach and holding-up of different set points (control loop) can be controlled by a PID Element. Hence transfer functions are needed for programming the microcontroller. With the resistance curves (figure 4) partial defined transfer functions can be realized. The gradients of heating and cooling curves can be calculated and defined for every point of the hysteresis. In order to simplify the algorithms it is possible to program just two linear lines with gradients corresponding to the middle part of the simulated hysteresis.

The upper line (heating trend) as well as the lower line (cooling trend) can be converted by Laplace transformation to transfer functions. The control loop (figure 5) can be realized as a microcontroller unit sending a steering signal to a current source. The source sets the current in coherence to the controller's signal. The voltage signal is converted to a digital signal, which is calculated to the corresponding resistance value. The PID controller operates with trend lines mentioned above which change by heating or cooling conditions. After system boot and self sensing (in order to detect base resistance data) the heating control loop is activated. If the set point is exceeded, the system changes to cooling condition, that means the heating transfer function is replaced by the cooling trend. Corresponding results are published by the authors within another publication [7]

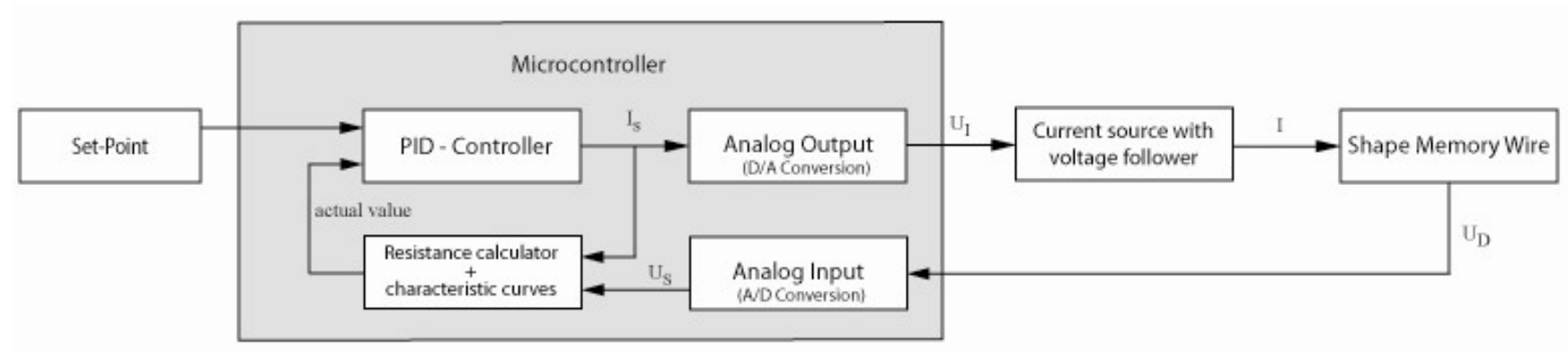

Fig. 5. Control loop of SMA actuator 


\section{Fatigue of shape memory alloys}

Unfortunately shape memory alloy actuators do not work completely without functional fatigue. Contraction and decompression velocities, as well as the maximal stroke, depend mainly on the number of activation cycles. Experimental analysis by the authors over 5.000 activations reveal that there are logarithmical coherences between actuator behavior and working cycles [8]. Other researches show that the boundary conditions, like the surrounding

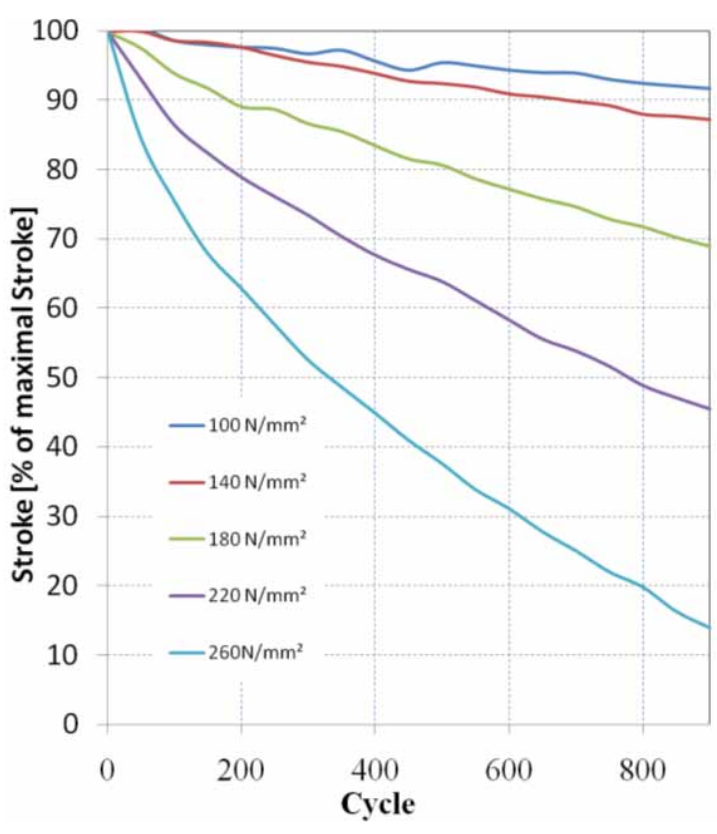

Fig. 6. SMA wire fatigue curves

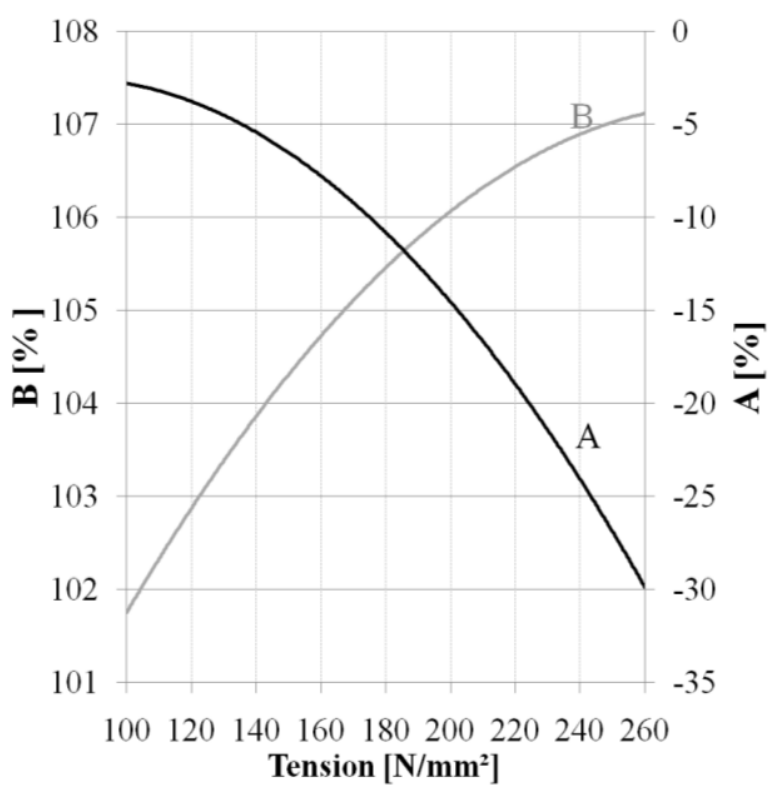

Fig. 7. Interpolated factors $A_{f}$ and $B_{f}$ over preload

medium or loads effect the fatigue of SMAs [9]. For correct control of shape memory systems, a regulating system has to count the driven cycles, and change the transfer functions in according functional fatigue. Hence algorithms calculating fatigue behavior are needed. Because of the varying quality of NiTi material a correct theoretical method for modeling the fatigue behavior is nearly impossible to develop. Though, an empirical approach in this field by testing and collecting data under different boundary conditions is expedient. The description of material behavior can be made (in similarity to fatigue analysis done for other metal based materials) by regarding a cycle depended behavior as experiments delivered in figure 6. Fatigue curves, for different preloads, are shown against the cycle number. It is obvious that the greatest change appears during the early cycles.

On a closer look for the first cycles the experimental results seem to be fatal for actuation. The fatigue of the high preloaded wires leads to the conclusion that SMAs are not suitable for mechatronical systems. Generally SMAs can be used for few high-loaded cycles or many cycles by low preloads. Precisely regarded, the fatigue depends not only on loads, but also on alloy type. As proved by J. Uchil [10] there are coherences between recoverable strain and heat-treatment. For closer examination and completion of the simulated behavior, continuative analysis in dependence of different alloy types and thermomechanical treatments have to be made. For development of a fatigue simulation and interpolation of such curves, a model for cycle and preload-dependant activation behavior has been created. 


$$
\begin{gathered}
\text { Maximal Stroke }=S_{\max }=A \cdot \ln (n)+B \\
\text { with: } \\
\begin{array}{c}
A=A_{100} \cdot A_{f}(\sigma) \\
B=B_{100} \cdot B_{f}(\sigma)
\end{array}
\end{gathered}
$$

In equation 6 the variable ' $n$ ' is the cycle number. The factors A and B depend on mechanical preload and have to be calculated from a database which was collected in experiments. The benchmark is chosen at $100 \mathrm{~N} / \mathrm{mm}^{2}$ which equals an average restoring force in macroscopical SMA applications using NiTi wires. In order to interpolate a fatigued stroke, the numerical simulation calculates the maximal stroke at first cycle. In the next step, the empirical tested curves for $A_{f}$ and $B_{f}$ stored in the numerical simulation are used to calculate the maximal stroke in by multiplication with the benchmark-factors.

A simulation of fatigue behavior can be done for all boundary conditions concerning loads, activation current and material type. Figure 8 exemplary shows a simulated activation of the first and the 1000st cycle.

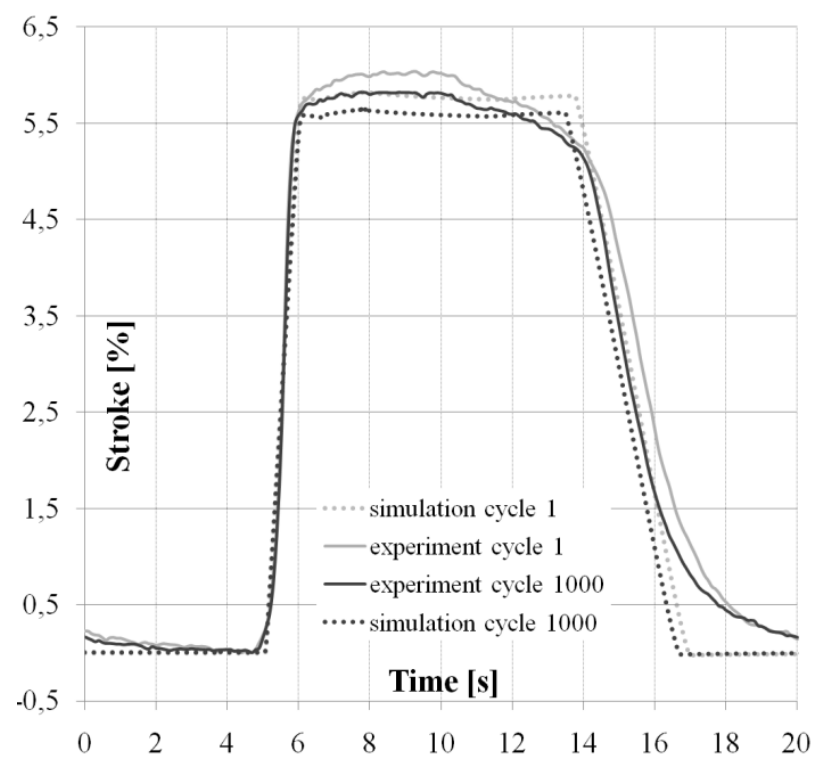

Fig. 8. Simulated and tested cycles in comparison

\section{Conclusions}

Analysis of SMA's electrical resistance revealed that a feedback can be used for controlling positions. Furthermore it is possible to include resistance calculation algorithms in the numerical model for activation, so this model can support a constructing engineer in system development. This enhances the upgrade possibilities for shape memory driven systems. One of the major problems concerning SMA actuators, the fatigue, can also be simulated in the numerical model. So engineers can calculate the life cycle of NiTi actuators.

\section{Acknowledgment}

The authors acknowledge funding by the DFG through SFB research centre 459 (Project B6) (Shape memory technology). 


\section{References}

[1] K.Worden,W.A. Bullough, J. Haywood, Smart Technologies (World Scientific, London, GB, 2003), p. 109, and p. 126

[2] L. Ölschläger, Nummerische Modellierung des Akrivierungsverhaltens von Formgedächtnisaktoren am Beispiel eines Schrittantriebes, (Shaker Verlag, Aachen, D, 2004)

[3] E. G. Welp, S. Langbein, „Neue Ansätze zur Baukastensystematik von Formgedächtnisaktoren“

VDI Berichte zur VDI-Tagung Mechatronik 2007, Innovative Produktentwicklung. Wiesloch 23./24.05.07. VDI-Bericht Nr.1971, p. 417-433

[4] K.Ikuta, M.Tsukamoto, S.Hirose, "Shape memory actuator system with electrical resi stance feedback and application for active endocope, IEEE Int. Conf. Robotics and Automation, Philadelphia, PA, USA, 1988, p. $427-430$

[5] R. Mukherjee, T. Christian, R. Thiel, "An actuation system for the control of multiple shape memory alloy actuators”, Sensors and Actuators A55, 1996, p. 185-192

[6] C.Liang, C.A. Rogers, "One-Dimensional Thermomechanical Costitutive Relations for Shape Memory Materials", Journal of intelligent material systems and structures, Vol 1, 1990

[7] H. Meier, A. Czechowicz, S. Dilthey, "Regeln von Formgedächtnislegierungen mit Widerstandsrückkopplung” Mechatronik F+M, 11/12 2008, 24-27, Hanser Verlag, München

[8] S. Dilthey, A. Czechowicz; - not published so far.

[9] H.Tobushi, T. Nakahara, Y. Shimeno, T. Hashimoto, "Low-Cycle Fatige of NiTi Shape Memory Alloy and Formulation of Fatigue Life", Journals of Engineering Materials and Technology, April 2000, Vol. 122 /p. 186 191

[10] J . Uchil, K. Mahesh, K. Kumara, "Electrical resistivity and strain recovery studies on the effect of thermal cycling under constant stress on R-phase in NiTi shape memory alloy", Physica B 324, 2002, p. 419-428 